\title{
Laser arc sound signal processing and welding status recognition based
}

\section{on geometric learning}

\author{
HUA Liang $^{\mathrm{a}^{*}} \quad$ ZHENG Chang-Wei $^{\mathrm{b}} \quad$ GU Ju-Ping ${ }^{\mathrm{c}} \quad$ LIU Yu-Qing $^{\mathrm{d}}$
}

\author{
School of electrical engineering , Nantong University, Nantong, Jiangsu Province, China \\ ahualiang@ntu.edu.cn, b1224746617@qq.com, cogu.jp@ntu.edu.cn, d328497196@qq.com
}

Keywords: wavelet threshold; Laser arc welding; multi-weight neural network; Feature extraction

Abstract. Laser arc sound signal hides welding status information. It is a great significance to control the welding quality. This paper builds the laser welding of $\mathrm{B} \& \mathrm{~K}$ company and four channels input sound and vibration analyzer. Using wavelet base which is $\mathrm{db} 4$, combining different methods to decompose laser arc sound signal. These methods include hard threshold, soft threshold and double threshold double factors. The results show that choosing double threshold double factor has the highest SNR. After processing, 1024 consecutive arc sound signal sampling points are selected as a sample, and features are extracted in time domain and frequency domain. Thirty samples are respectively selected from three welding conditions including complete penetration, incomplete penetration and welding wear, which constitute training samples. Twenty samples are respectively selected from three welding conditions, which constitute test samples. test samples are respectively identified by probabilistic neural network(PNN) and multi-weights neural network(MWNN). Results show that the whole recognition rate of multi-weight neural network is higher than the whole recognition rate of the probability neural network.

\section{Introduction}

With the development of industrial production, laser welding technique plays an important role in actual application. In laser arc welding process, the volume expansion and excessive drip oscillation will produce great voice, which contains mixed voice ${ }^{[1]}$. According to the variation of arc sound, experienced welders can roughly determine the quality of welding in the practical work ${ }^{[2,3]}$ by personal experience. Thus the arc sound signal indeed contains important information of welding status ${ }^{[4]}$. It has achieved certain results to research on acoustic signal of welding process in the country. Qiang Chen ${ }^{[5]}$ and Yaowen Wang study plasma arc welding perforation behavior, who are from Tsinghua University. The research further suggests that the low frequency component of sound signal contains information of welding penetration status. Jinglei $\mathrm{Liu}^{[6]}$, a scholar at East China University of Science and Technology and Yanbin Chen,a scholar at Harbin industrial university, study the correlation of laser welding sound signal and penetration status in the process of laser welding, and establish the relationship between the state of welding penetration and sound signal through artificial neural network. Ladislav Grad and Janez Grum study laser welding sound signal abroad, who draw a conclusion that the irregular change of welding arc sound related to the instability of the welding process ${ }^{[7]}$. But collected arc sound signal contains some noises in the actual situation, so it becomes the first problem to deal with laser arc sound signals.

In this paper, laser arc sound signal is used as the object of study. The collected sound signal is processed by different threshold method. The characteristics of arc sound signal are extracted from two aspects: time domain and frequency domain. In pattern recognition, the extracting characteristic values are selected as input. Three welding states are selected as output. The probabilistic neural network (PNN) and the multi-weight neural network (MWNN) mapping models are obtained by constructing the training sample. Then the trained neural network models are used to classify the 
welding conditions of test samples.

\section{Arc sound signal acquisition}

Laser arc sound signal acquisition system is shown in Fig 1. It mainly includes PULSE labshop software, sound sensor of B\&K company, four channels input sound and vibration analyzer, welding equipment etc.

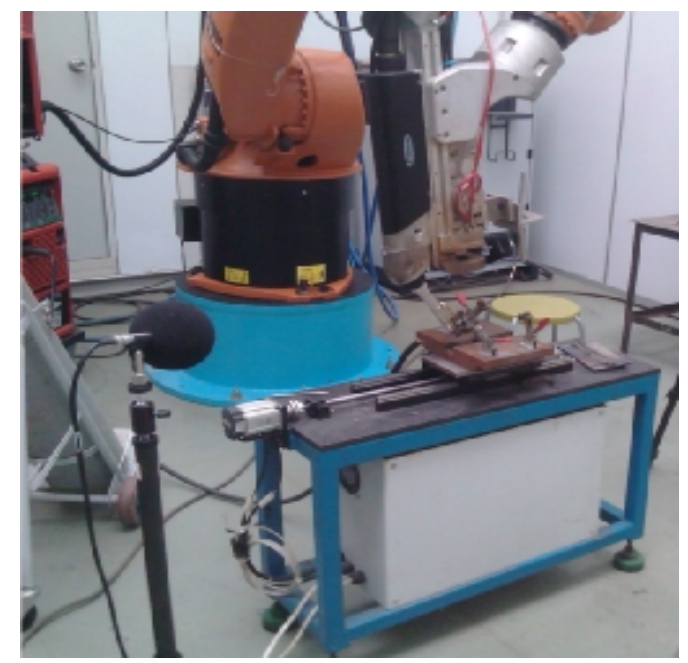

Fig 1. Laser arc sound signal acquisition devices

Before the acquisition of signal, it needs to configure the IP address of the PC and connect 3050A-040 LAN-XI sound and vibration analyzer. After setting PULSE labshop software, the whole set of process is shown in Fig 2.

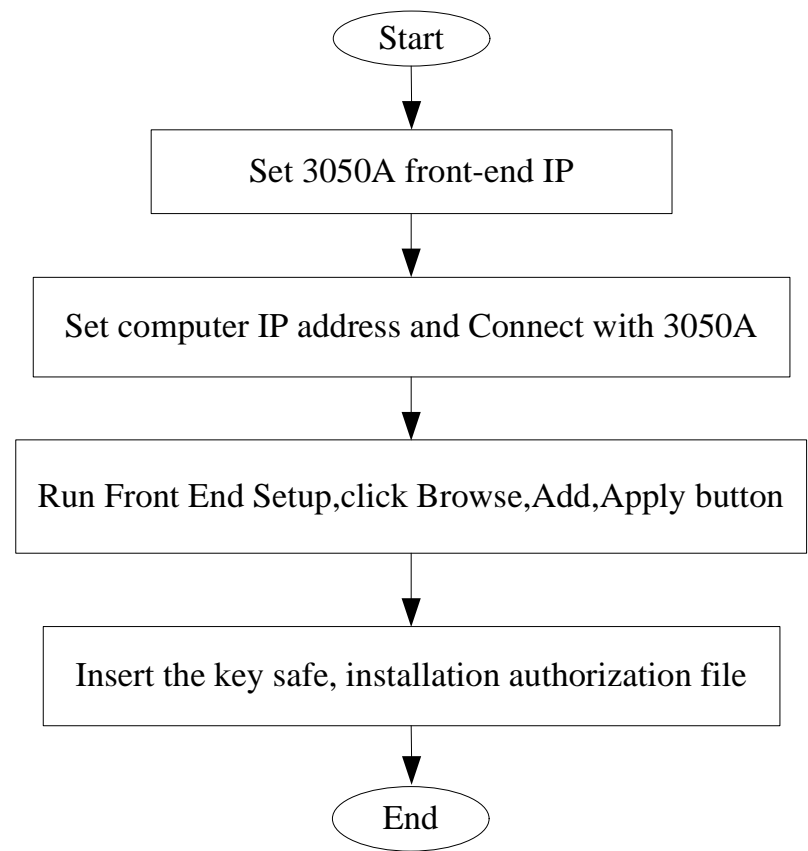

Fig 2. Set before the acquisition

In the welding process, laser welding equipment model is TLF1500. Weldment type is HSLA-115 low carbon high strength alloy steel. Their sizes are respectively $200 \mathrm{~mm} \times 100 \mathrm{~mm} \times 6 \mathrm{~mm}$, $200 \mathrm{~mm} \times 100 \mathrm{~mm} \times 8 \mathrm{~mm}$ and $200 \mathrm{~mm} \times 100 \mathrm{~mm} \times 10 \mathrm{~mm}$. Incomplete penetration experiment is conducted with $6 \mathrm{~mm}$ steel plate. Complete penetration experiment is conducted with $8 \mathrm{~mm}$ steel plate. Welding wear experiment is conducted with $10 \mathrm{~mm}$ steel plate. Acquisition of laser arc sound signal is shown in Fig 3. 


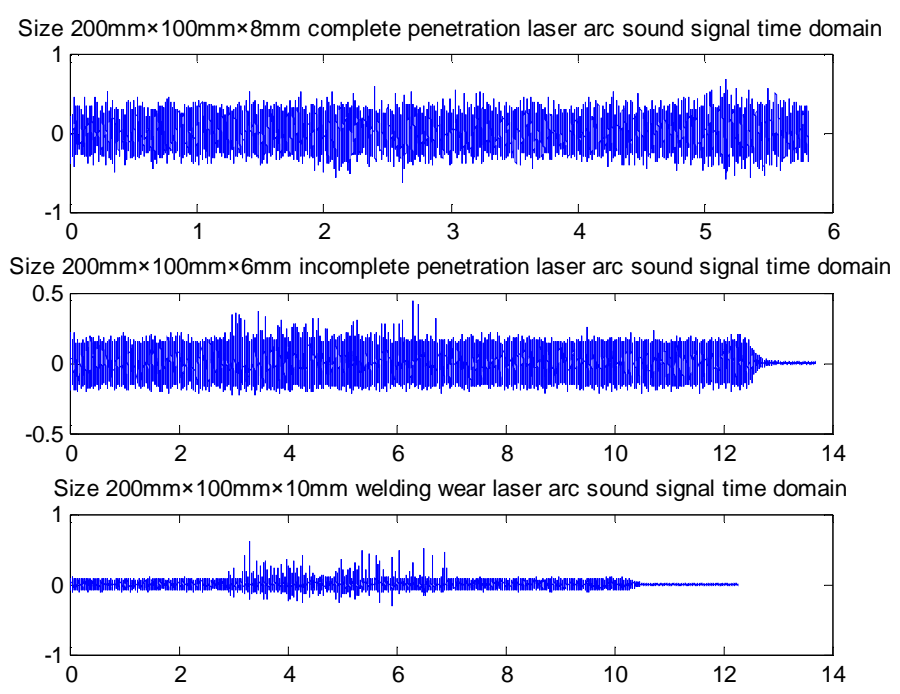

Fig 3. Time domain of arc sound signal under different welding state

\section{De-noising method}

In the welding process, arc sound signal are mixed in the noise of shielding gas including mechanical noise and electromagnetic noise. In order to better study of arc sound signal, the collected arc sound signals are processed by wavelet threshold to reduce noise, which wavelet threshold adopt different threshold method.

Double threshold double factor function

According to energy distribution of signal and noise in wavelet domain ${ }^{[8]}$, Donoho puts forward hard threshold ${ }^{[9,10]}$ and soft threshold method to reduce noise. When Wavelet transform coefficient is less than the threshold in double threshold double factor function, it is different from traditional threshold functions, which is based on Lagrange Interpolation theorem ${ }^{[12]}$.

Double threshold double factor function defines the first threshold $\lambda_{1}$ and the second threshold $\lambda_{2}\left(0<\lambda_{2}<\lambda_{1}\right.$, in addition, $\left.\lambda_{2}=\beta \lambda_{1}\right)$, which describes deviation factor $\alpha$ and threshold factor $\beta$, Where two factors meet the conditions: $0 \leq \alpha \leq 1,0 \leq \beta \leq 1$. It can achieve optimal de-noising effects by changing the value of $\alpha$ and the value of $\beta$. Double threshold double factor ${ }^{[8]}$ function is defined as follows:

$$
\bar{d}_{j k}=\left\{\begin{array}{ll}
\operatorname{sgn}\left(d_{j k}\right) \cdot\left(\left|d_{j k}\right|-\lambda_{1}+\alpha \lambda_{1} / e^{(1-\alpha)}\right) & \left|d_{j k}\right|>\lambda_{1} \\
\operatorname{sgn}\left(d_{j k}\right) \cdot\left(\alpha \lambda_{1} / e^{(1-\alpha)} \cdot t\right) & \left|\lambda_{2} \leq d_{j k} \leq \lambda_{1}\right| \\
0 & \left|d_{j k}\right|<\lambda_{2}
\end{array} .\right.
$$

\section{De-noising performance.}

In order to verify double threshold double factor function better than the traditional threshold function in terms of de-noising aspect, where signal-to-noise ratio and root mean square error are compared.

Here, $x(i)$ is the original signal, $\hat{x}(i)$ is the signal after de-noising, $\mathrm{N}$ is the length of the signal. The larger the SNR and the smaller RMSE, the better de-noising effect. The results are shown in table 1 . 
Table 1 Root mean square error and signal to noise ratio under different threshold function

\begin{tabular}{|c|c|c|c|c|}
\hline & & $\begin{array}{c}\text { Hard } \\
\text { threshold }\end{array}$ & $\begin{array}{c}\text { Soft } \\
\text { threshold }\end{array}$ & $\begin{array}{c}\text { Double threshold and } \\
\text { double factor }\end{array}$ \\
\hline $\begin{array}{c}\text { Height 6mm } \\
\text { incomplete penetration }\end{array}$ & SNR(dB) & 6.2902 & 6.2548 & 19.4335 \\
\cline { 2 - 5 } RMSE & 0.0245 & 0.0246 & 0.0054 \\
\hline $\begin{array}{c}\text { Height 8mm } \\
\text { complete penetration }\end{array}$ & SNR(dB) & 24.8399 & 24.8292 & 37.4885 \\
\cline { 2 - 5 } & RMSE & 0.0087 & 0.0088 & 0.002 \\
\hline Height 10mm & SNR(dB) & 6.0192 & 5.9268 & 19.5432 \\
\cline { 2 - 5 } welding wear & RMSE & 0.0125 & 0.0126 & 0.0026 \\
\hline
\end{tabular}

The de-noising ability of double threshold and double factors is better than traditional threshold function through the observation in table 1 . This contributes to the following feature extraction.

\section{Feature extraction}

\section{Short time window technique}

Arc sound signal is changed over time, but it can be seen as a process of quasi steady state in a short time. So it can use smooth process of processing method and theory for the short-term treatment of arc sound signal. Every short period is called a "frame analysis", where window function used is hamming window ${ }^{[14]}$ in this paper.

\section{Time domain and frequency domain features}

Short-time energy

There is a significant difference in the signal energy of different welding state. Short-time energy analysis of arc sound signal reflects amplitude changes. Short-time energy ${ }^{[15,16]}$ function is defined as follows:

$$
E_{n}=\sum_{m=-\infty}^{\infty}[x(m) \omega(n-m)]^{2}=\sum_{m=-\infty}^{\infty} x^{2}(m) h(n-m)=x^{2}(n) h(n) .
$$

Where $x(m)$ is arc sound signal. $E_{n}$ is short-time energy.

Short-time average magnitude

Short-time energy calculate the squares of the signal samples. But short-time average magnitude is measured by calculating the sum of absolute value change in arc sound signal. Short-time average magnitude function ${ }^{[17]}$ is defined as follows:

$$
M_{n}=\sum_{m=-\infty}^{\infty}\left|x(m) \omega(n-m)=\sum_{m=n}^{n+N-1}\right| x_{\omega}(m) \mid
$$

Where $x(m)$ is arc sound signal. $M_{n}$ is short-time average magnitude.

Short-time average zero-crossing rate

Short-term average zero crossing rate calculate the number of sample values changes sign in each frame. Short-time average zero-crossing rate function ${ }^{[15,16]}$ is defined as follows:

$$
Z_{n}=\frac{1}{2} \sum_{m=n}^{n+N-1} \mid \operatorname{sgn}\left[x_{\omega}(m)-\operatorname{sgn}\left(x_{\omega}(m-1)\right)\right] .
$$

Where: $\operatorname{sgn}[x(n)]=\left\{\begin{array}{cc}1 & x(n) \geq 0 \\ -1 & x(n)<0\end{array}\right.$.

Short-time zero-energy ratio

Short-time zero-energy ratio is combined with the short-term energy and zero crossing rate. The ratio of zero crossing rates to short-time energy at the same time. Short-time zero-energy ratio function ${ }^{[15,16]}$ is defined as follows: 


$$
Z E R_{n}=Z_{n} / E_{n}=\frac{\sum_{m=n}^{n+N-1}\left|\operatorname{sgn}\left[x_{\omega}(m)-\operatorname{sgn}\left(x_{\omega}(m-1)\right)\right]\right|}{2 \sum_{m=n}^{n+N-1} x_{\omega}{ }^{2}(m)} .
$$

Where $Z_{n}$ is Short-time average zero-crossing rate. $E_{n}$ is Short-time energy.

\section{Short-time power spectrum}

Power spectrum analysis also adopts short-term analysis. It is based on Fourier Transform. Short-time Fourier transform ${ }^{[18]}$ of signals is defined as follows:

$$
X_{n}\left(e^{j \omega}\right)=\sum_{m=-\infty}^{\infty} x(m) \omega(n-m) e^{-j \omega m}
$$

The method of calculating power spectrum uses discrete Fourier Transform. Short-time power spectrum is actually the square of Fourier transform magnitude, where describes as $P_{n}$.

This paper conducts fifty percent overlapping of arc sound signal framing by using Hamming window. The window length is $1024 . E_{n}, M_{n}, Z_{n}, Z E R_{n}, P_{n}$ were calculated in three different welding state. The results are shown in table 2 .

Table 2 The eigenvalues of the different welding conditions

\begin{tabular}{|c|c|c|c|c|c|}
\hline & $E_{n}$ & $M_{n}$ & $Z_{n}$ & $Z E R_{n}$ & $P_{n}$ \\
\hline $\begin{array}{c}\text { Height 6mm } \\
\text { incomplete penetration }\end{array}$ & 4.2892 & 0.0426 & 29 & 6.7612 & 36.4267 \\
\hline $\begin{array}{c}\text { Height 8mm } \\
\text { complete penetration }\end{array}$ & 1.2049 & 0.0235 & 142 & 117.8521 & 30.9126 \\
\hline $\begin{array}{c}\text { Height 10mm } \\
\text { welding wear }\end{array}$ & 0.3790 & 0.0127 & 177 & 467.0185 & 25.8896 \\
\hline
\end{tabular}

\section{Welding states recognition}

Neural network ${ }^{[19,20]}$ is a complex network system which is formed by a large number of simple processing units. $E_{n}, M_{n}, Z_{n}, Z E R_{n}, P_{n}$ are selected as input, incomplete penetration, complete penetration and welding wear are selected as target output. Through the training samples, the probabilistic neural network (PNN) and the multi-weight neural network (MWNN) classifier are respectively established to recognize the welding conditions of the arc sound signal.

\section{Probabilistic neural network}

PNN is a mentor learning feed-forward neural network, which generally consists of input layer, hidden layer and output layer. It uses the Bayes minimum risk rule for classification, which is shown in Fig 4. 


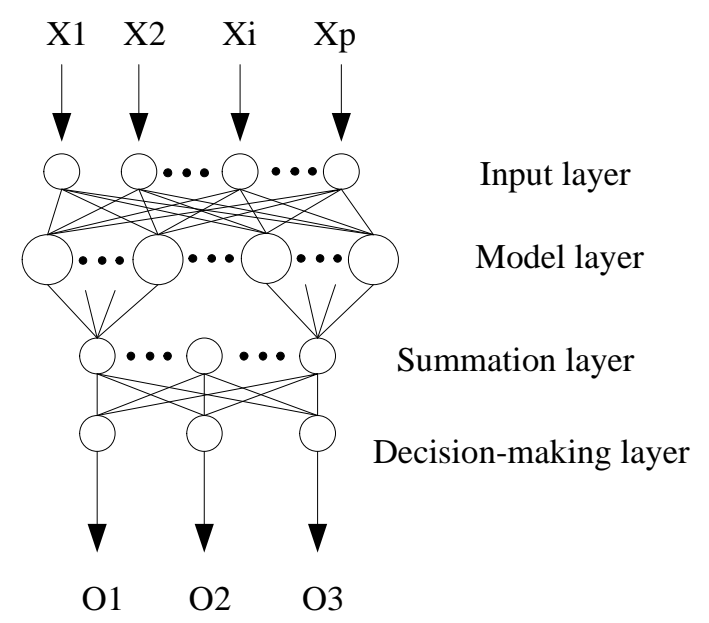

Fig 4 Probabilistic neural network basic structure

Input layer pass input sample to model layer nodes. Model layer conducts weighted summation through weight. Summation layer accumulates the samples input from pattern layer. Decision-making layer selects the maximum probability density of the neuron as the output.

Results and analysis

In the experiment, 1024 data were used as a sample. 50 samples were selected respectively from three different welding conditions. The training sample is made up of 30 samples from three different welding conditions. The rest is as the test sample. So the number of training sample is 90 . The total number of test samples is 60. The experimental results is shown in figure 6 and figure 7 . "1" represents complete penetration, "2" represents incomplete penetration, "3" represents welding wear.
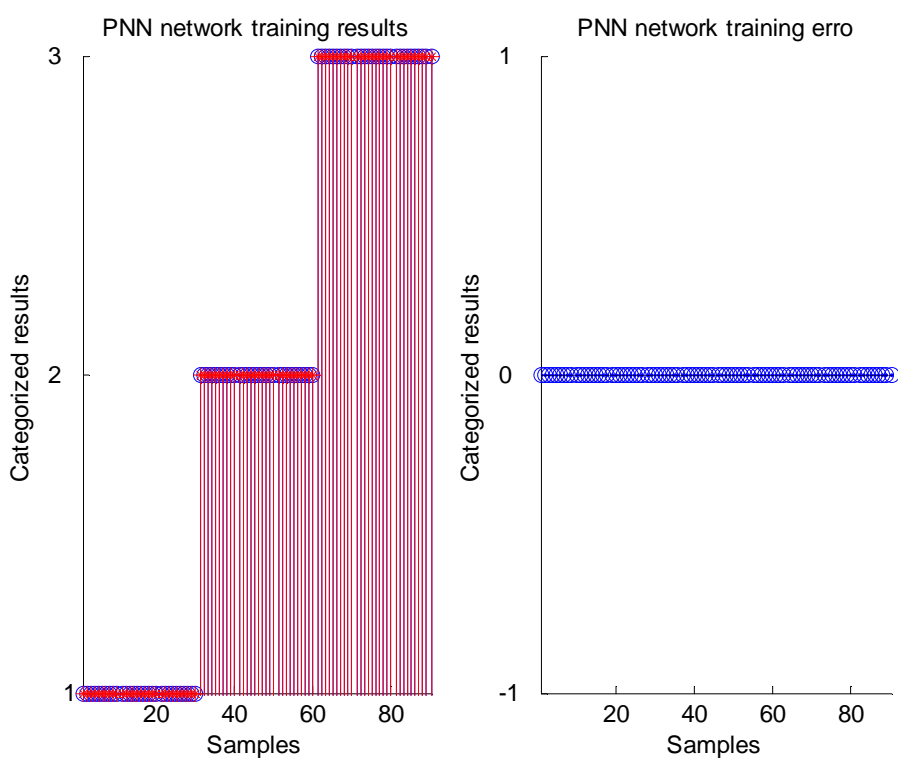

Fig 5 PNN classification results and error of training samples 


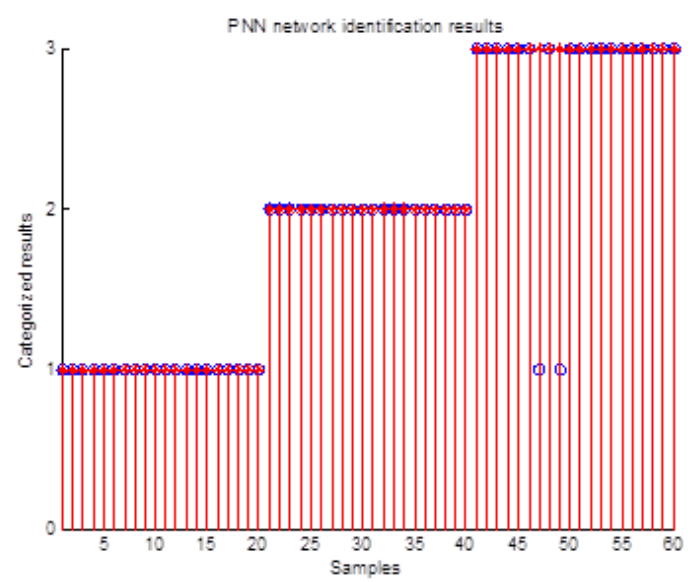

Fig 6 PNN network identification results

In Fig 5, Training samples error of PNN is zero, where "1" represents complete penetration. "2" represents incomplete penetration. "3" represents welding wear. Classification results of test samples are shown in Fig 6, where recognition rate of complete penetration is $100 \%$, recognition rate of incomplete penetration is $100 \%$, recognition rate of welding wear is $90 \%$, which has two false identification as complete penetration. The whole test samples recognition rate is $96.67 \%$.

\section{Geometric learning}

Basic principle of geometry learning

Geometric study is based on the similar samples in the best cover of the specific spatial distribution as a target. All samples of similar things were in the area of an irregular connected, according to certain rules to establish a high dimensional complex geometry to cover the area through the network learning. If samples were identified in the enclosed area, these samples were identified correctly. On the contrary, if samples were identified outside the closed area, these samples refused to identify.

This paper used three weights of neurons ${ }^{[21]}$, which expressed as follows:

$$
\left\{\begin{array}{l}
Y=f\left[\varphi\left(w_{1}, w_{2}, w_{3}, x\right)-\theta\right] \\
\varphi\left(w_{1}, w_{2}, w_{3}, x\right)=\left\|X-\psi_{\left(W_{1}, W_{2}, W_{3}\right)}\right\| \\
\Psi_{\left(W_{1}, W_{2}, W_{3}\right)}=\left\{Y \mid Y=\alpha_{2}\left[\alpha_{1} W_{1}+\left(1-\alpha_{1}\right) W_{2}\right]+\left(1-\alpha_{2}\right) W_{3}\right\}
\end{array} .\right.
$$

Where $\alpha_{1} \in[0,1], \alpha_{2} \in[0,1], \varphi_{(w 1, w 2, w 3)}$ represented three points(w1, w2, w3)to form a triangular region. $\varphi(w 1, w 2, w 3, x)-\theta \leq 0$ described as $\varphi_{(w 1, w 2, w 3)}$ and hypersphere(radius of $\theta$ )determine coverage after topology product.

Built steps of geometric learning

The training sample sets are $X=\left\{X_{1}, X_{2}, \mathrm{~L} X_{n}\right\}$ after some sort of fault feature extraction. $\mathrm{N}$ is the number of sample points. $X_{i}=\left(x_{1}^{i}, x_{2}^{i}, \mathrm{~L}, x_{m}^{i}\right)$ is one of samples, where $\mathrm{m}$ is the dimension of samples points, $\rho_{X \varphi i}$ is the euclidean distance of the point $X$ and the space $\varphi_{i}, \alpha_{1} \in[0,1]$, $\alpha_{2} \in[0,1]$, built steps of geometry learning ${ }^{[21]}$ are as follows:

Step 1: First of all find out point-to-point euclidean distance in the training sample $X$, and record the minimum euclidean distance of two points as $P_{11}, P_{12}$. In addition to these two points, one of all remaining points to the sum of the distance of these two points is minimum, and the point is not collinear with point $P_{11}$ and $P_{12}$, which noted as $P_{13}$, get three points form a closed area $\left(\Delta P_{11} P_{12} P_{13}\right)$, which noted $\operatorname{as} \varphi_{1}$. And cover the area with a neurons $P^{S i 3}$, the scope of coverage is ${ }^{[22]}$ : 


$$
\begin{aligned}
& P_{1}=\left\{X \mid \rho_{X \psi_{1}} \leq \theta, X \in R^{n}\right\} . \\
& \psi_{1}=\left\{Y \mid Y=\alpha_{2}\left[\alpha_{1} P_{11}+\left(1-\alpha_{1}\right) P_{12}\right]+\left(1-\alpha_{2}\right) P_{13}\right\}
\end{aligned}
$$

Step 2: For the former constructed geometry $P_{1}$, whether the remaining points were contained by the geometry $P_{1}$. If within the scope of the body cover, then eliminate this point. For those sample points outside the body, according to the method of step one, finding one point to the euclidean distance of these three points is minimum, noted the point as $P_{21}$. After that calculated separately these three points $\left(P_{11}, P_{12}, P_{13}\right)$ to the euclidean distance of $P_{21}$, and took the minimum value and minor value. Then remembered as $P_{22}, P_{23}$ again. So we got the second enclosed area $\left(\Delta P_{21} P_{22} P_{23}\right)$, which was noted $\operatorname{as} \varphi_{2}$. To cover the other area with a neurons $P^{S i 3}$, the scope of coverage is ${ }^{[22]}$ :

$$
\begin{aligned}
& P_{2}=\left\{X \mid \rho_{X \psi_{2}} \leq \theta, X \in R^{n}\right\} . \\
& \psi_{2}=\left\{Y \mid Y=\alpha_{2}\left[\alpha_{1} P_{21}+\left(1-\alpha_{1}\right) P_{22}\right]+\left(1-\alpha_{2}\right) P_{23}\right\} .
\end{aligned}
$$

Where $\rho_{X \varphi 2}$ is the euclidean distance of the point $X$ and the space $\varphi_{2}$.

Step 3: First remove all the points of within the cover scope of $P_{i-1}$. Then calculated the distance of three points of $\varphi_{i-1}$ to point $P_{i-1}$, selected two short distances and noted two points as $P_{i 2}, P_{i 3}$. Got the coverage area $\left(\Delta P_{i 1} P_{i 2} P_{i 3}\right)$, which noted $\operatorname{as} \varphi_{i}$. To cover the area with a neurons $P^{S i 3}$, the scope of coverage is ${ }^{[22]}$ :

$$
\begin{aligned}
P_{i} & =\left\{X \mid \rho_{X \psi_{i}} \leq \theta, X \in R^{n}\right\} . \\
\psi_{i} & =\left\{Y \mid Y=\alpha_{2}\left[\alpha_{1} P_{i 1}+\left(1-\alpha_{1}\right) P_{i 2}\right]+\left(1-\alpha_{2}\right) P_{i 3}\right\} .
\end{aligned}
$$

Step 4: Repeated to operate step three for all the sample points, until handling all the sample points. Eventually built $\mathrm{M}$ geometries and their union noted as $\Omega$.

$$
\Omega=\bigcup_{j=1}^{M} \psi_{i} .
$$

Experimental results and analysis

Training samples and test samples of multi-weights neural network are the same as PNN.Calculate the smallest distance of test samples to the space geometry $\Omega_{i}(i=1,2,3)$, the results are shown in table 3. To determine which type of welding status by the smallest distance. The results are shown in figure 8 , in the picture, "1" represents complete penetration, "2" represents incomplete penetration, "3" represents welding wear. 
Table 3 the smallest distance $\rho_{i}$ of test samples to the space geometry $\Omega_{i}(i=1,2,3)$

\begin{tabular}{|c|c|c|c|c|c|c|c|c|c|c|c|}
\hline $\begin{array}{c}\text { Num } \\
\text { ber }\end{array}$ & $\rho_{1}$ & $\rho_{2}$ & $\rho_{3}$ & $\begin{array}{c}\text { Num } \\
\text { ber }\end{array}$ & $\rho_{1}$ & $\rho_{2}$ & $\rho_{3}$ & $\begin{array}{c}\text { Num } \\
\text { ber }\end{array}$ & $\rho_{1}$ & $\rho_{2}$ & $\rho_{3}$ \\
\hline 1 & 0.0096 & 2.1643 & 2.5040 & 21 & 1.9406 & 0.0409 & 0.5408 & 41 & 2.6192 & 1.0828 & 0.0528 \\
\hline 2 & 0.0402 & 1.8891 & 2.3300 & 22 & 1.8552 & 0.0027 & 0.8127 & 42 & 2.7308 & 1.3572 & 0.0018 \\
\hline 3 & 0.2124 & 1.7537 & 2.2106 & 23 & 1.7853 & 0.0044 & 1.0326 & 43 & 2.7077 & 1.2847 & 0.0003 \\
\hline 4 & 0.0278 & 2.3425 & 2.6615 & 24 & 1.8238 & 0.0053 & 0.7094 & 44 & 2.6805 & 1.2213 & 0.0002 \\
\hline 5 & 0.0067 & 2.4712 & 2.7556 & 25 & 1.7239 & 0.0407 & 1.1255 & 45 & 2.6264 & 1.1372 & 0.0043 \\
\hline 6 & 0.0762 & 2.3166 & 2.6367 & 26 & 1.8433 & 0.0023 & 0.6526 & 46 & 2.6273 & 1.1095 & 0.0433 \\
\hline 7 & 0.1679 & 2.5564 & 2.9109 & 27 & 1.7763 & 0.0014 & 0.9738 & 47 & 2.8510 & 1.5513 & 0.2066 \\
\hline 8 & 0.0160 & 2.4034 & 2.7082 & 28 & 1.7499 & 0.0098 & 0.9036 & 48 & 2.7964 & 1.4723 & 0.0245 \\
\hline 9 & 0.0298 & 1.9448 & 2.3388 & 29 & 1.9350 & 0.0208 & 0.5116 & 49 & 2.8169 & 1.5046 & 0.1038 \\
\hline 10 & 0.0191 & 1.9880 & 2.3691 & 30 & 2.0812 & 0.0570 & 0.2415 & 50 & 2.7363 & 1.3646 & 0.0010 \\
\hline 11 & 0.0035 & 2.4939 & 2.7908 & 31 & 1.9148 & 0.0225 & 0.5442 & 51 & 2.4897 & 0.7921 & 0.0688 \\
\hline 12 & 0.1203 & 2.2323 & 2.5761 & 32 & 1.7116 & 0.0249 & 1.0826 & 52 & 2.7324 & 1.3365 & 0.0245 \\
\hline 13 & 0.0694 & 1.8229 & 2.3012 & 33 & 1.7502 & 0.0070 & 0.9392 & 53 & 2.7503 & 1.3868 & 0.0020 \\
\hline 14 & 0.0238 & 2.2109 & 2.5658 & 34 & 1.8511 & 0.0005 & 0.8118 & 54 & 2.7461 & 1.3863 & 0.0063 \\
\hline 15 & 0.0612 & 2.3822 & 2.6957 & 35 & 2.1284 & 0.0204 & 0.1369 & 55 & 2.7136 & 1.3278 & 0.0004 \\
\hline 16 & 0.1424 & 2.4894 & 2.8493 & 36 & 1.8125 & 0.0043 & 0.9032 & 56 & 2.6524 & 1.1783 & 0.0013 \\
\hline 17 & 0.2286 & 2.6186 & 2.9554 & 37 & 1.8935 & 0.0014 & 0.5823 & 57 & 2.7257 & 1.3452 & 0.0021 \\
\hline 18 & 0.2334 & 2.5190 & 2.8776 & 38 & 2.1193 & 0.1232 & 0.1632 & 58 & 2.7282 & 1.3452 & 0.0023 \\
\hline 19 & 0.3592 & 2.6713 & 2.9897 & 39 & 1.8112 & 0.0062 & 0.8582 & 59 & 2.6903 & 1.2583 & 0.0013 \\
\hline 20 & 0.0027 & 2.5360 & 2.8268 & 40 & 1.8496 & 0.0014 & 0.8262 & 60 & 2.7303 & 1.3374 & 0.0182 \\
\hline
\end{tabular}

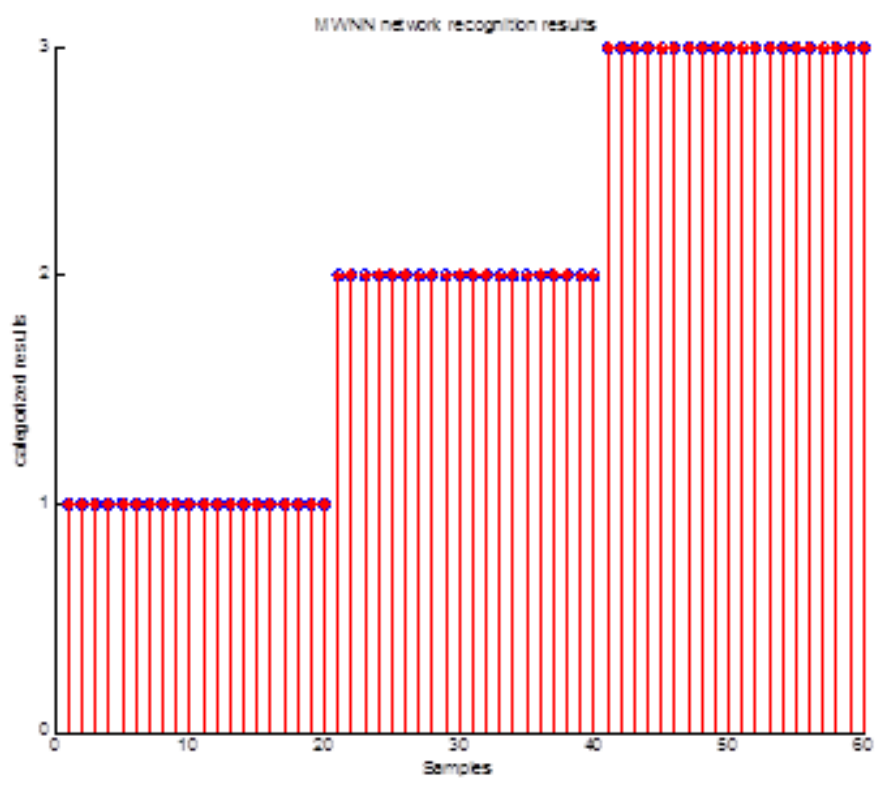

Fig 7 MWNN network recognition results

In Fig 7, "1" represents complete penetration that recognition rate is $100 \%$, "2" represents incomplete penetration that recognition rate is $100 \%, " 3$ " represents welding wear that recognition rate is $100 \%$. The whole recognition rate of MWNN is $100 \%$.

\section{Summary}

This paper denoises arc sound signal by combining different threshold methods. The results show that double threshold double factor de-noising ability is the best than two other methods by 
comparing the SNR and the RMSE.

1024 consecutive arc sound signal sampling points are selected as a sample. 50 samples were selected respectively from three welding conditions. selected 30 samples respectively from three welding conditions, which form training samples. The rest is as the test sample. the whole recognition rate of MWNN is $100 \%$. The wholel recognition rate of PNN is $96.67 \%$. So the whole recognition rate of $\mathrm{MWNN}$ is higher than $\mathrm{PNN}$.

\section{Acknowledgements}

This work was supported by the National Natural Science Foundation of China (61273024, 61305031), the Natural Science Foundation of Jiangsu Province (KB2012227), the "333 High-level Personnel Training Project" of Jiangsu Province, a Project Funded by the Priority Academic Program Development of Jiangsu Higher Education Institutions (PAPD), the "226 High-level Personnel Training Project” of Nantong city.

\section{References}

[1] Hu Lan. Penetration State Recognition of MIG Welding Based on Signal Characteristics of Arc Sound[D]. Harbin: Harbin University of Science and Technology, 2009.

[2] SAINI D, FLOYD S. An Investigation of Gas Metal Arc Welding Sound Signature for On-line Quality Cl [J]. Welding Journal, 1998, 77(4): 172-179.

[3] INOUE K, TAKAHASHI Y, ZHANG J. Measurement of Arc Sound with Bum-through in MAW Welding[J]. Welding International, 1993, 7(10): 770-775.

[4] MA Y Z, MA W B, QU M. Characteristics Analyzing and Parametric Modeling of the Arc Sound in CO2 GMAW for On-line Quality Monitoring[J]. China Welding, 2006, 15(2): 6-13.

[5] Yaowen Wang, Qiang Chen, Zhenguo Sun, etc. Acoustic Signal Sensor for The Behavior of Plasma Arc Welding[J]. Chinese Journal of Mechanical, 2001, 37(1): 53-56.

[6] Jinlei Liu, Yanbin Chen, Qinghong Xu. Correlation between The Acoustic Signal of Laser Welding and Welding Depth[J]. Transactions of the China Welding institution. 2006, 27(1): 72-80.

[7] LADISLIV G, JANEZ G, IVAN P. Feasibility Study of Acoustic for On-line Monitory in Short Circuit Gas Metal Arc Welding[J]. International Journal of Machine Toll \& Manufacture, 2004, 44: 551-561.

[8] Zhong Ren, Ying Liu, Guodong Liu, Zhen Huang. Improved Wavelet Dual Threshold Dual Factor Fnction De-noising[J]. Journal of Computer applications, 2013, 33(9): 2595-2598.

[9] DONOHO D L, JOHNSTONE IM. Adapting to Unknown Smoothness Vis Wavelet Shrinkage[J]. Journal of American Stat A ssoc, 1995, 12(90): 1200-1224.

[10] DONOHO D L, De-noising by Soft-thresholding[J]. IEEE Trans on IT, 1995, 41(3): 613-627.

[11] Weiqiang Zhang, Guoxiang Song. A New Threshold Function Based on Wavelet Domain Signal De-noising[J]. Journal of Xi'an Electronic and Science University, 2004, 31(2): 296-299.

[12] Lijun Gao. Lagrange Interpolation Method in The Application of Numerical Information Hiding and Analysis[J]. Bulletin of Science and Technology, 2012, 28(4): 8-10.

[13] Xiaoxia Guo, Huizhong Yang. A Kind of Improved Compromise Method of Soft Hard Threshold Wavelet De-noising[J]. CAA I Transactions on Intelligent Systems, 2008, 3(3): 222-225.

[14] Hang Hu. Speech Signal Processing[M]. Heilongjiang: Harbin Institute of Technology, 2000.5. [15] Xiongwei Zhang. Modern Speech Processing Technology and Application[M]. Beijing: China Machine Press, 2003. 8.

[16] Li Zhao. Speech Signal Processing[M]. Beijing: China Machine Press, 2003. 3.

[17] Zhixiang Wu, Xunli Wang, Zhanfeng Li, Bin Qiu, Liping Qiu. The Speech Recognition System Based on Short-time Average Magnitude and HMM Research[J]. Xi'an: Control and Instruments in Chemical Industry, 2013.6.

[18] Liming Zhu, Xinwen Liu, Binglin Zhong, Han Ding. Short-time Power Spectrum of Vibration 
Signal Time-frequency Two-dimensional Feature Extraction Methods and Applications[J]. Journal of Vibration Engineering, 2004, 17(4): 443-446.

[19] Kaili Zhou, Yaohong Kang. Neural Network Model and MATLAB Simulation Program Design[M]. Beijing: Tsinghua University Pres, 2005: 66-87.

[20] Yuanbin Hou, Jingyi Du, Mei Wang. Neural Network[M]. Xi'an:Xi'an University of Electronic Science and Technology Press, 2007: 15-24.

[21] Lai Wei, Feifei Xu, Shoujue Wang. A Multi-weight Neuron Network Coverage Construction Method[J]. Chinese journal of computers, 2012, 35(5): 1031-1037.

[22] Shoujue Wang, Yangyang Liu, Jiangliang Lai, Xingxing Liu. Biomimetic pattern recognition and multi-weight neuron[M]. National Defence Industry Press, Beijing, 2012. 